\title{
Plasma cholesterol lowering in an AngII-infused atherosclerotic mouse model with moderate hypercholesterolemia
}

\author{
ARÁNZAZU ROSSIGNOLI ${ }^{1}$, EMINA VORKAPIC ${ }^{2}$, ANDERS WANHAINEN $^{3}$, TOSTE LÄNNE $^{4}$, \\ JOSEFIN SKOGBERG ${ }^{1}$, ERIKA FOLESTAD ${ }^{1}$ and DICK WÅGSÄTER ${ }^{2}$
}

\author{
${ }^{1}$ Division of Vascular Biology, Department of Medical Biochemistry and Biophysics, Karolinska Institutet, 17177 Stockholm; \\ ${ }^{2}$ Division of Drug Research, Department of Medical and Health Sciences, Linköping University, 58183 Linköping; \\ ${ }^{3}$ Department of Surgical Sciences, Section of Vascular Surgery, Uppsala University, 75185 Uppsala; \\ ${ }^{4}$ Division of Cardiovascular Medicine, Department of Medical and Health Sciences, \\ Linköping University, 58183 Linköping, Sweden
}

Received September 15, 2017; Accepted February 22, 2018

DOI: $10.3892 /$ ijmm.2018.3619

\begin{abstract}
Atherosclerosis is the main underlying causes of cardiovascular disease. There is a well-established association between high blood cholesterol levels and the extent of atherosclerosis. Furthermore, atherosclerosis has been proposed to augment abdominal aortic aneurysm (AAA) formation. As patients with AAA often have parallel atherosclerotic disease and are therefore often on cholesterol-lowering therapy, it is not possible to fully address the independent effects of plasma cholesterol lowering (PCL) treatment on AAA. The present study investigated the effect of angiotensin II (AngII)-infusion in modestly hypercholesterolemic Ldlr- $^{-/}$Apo $b^{100 / 100}$ Mttp flox/flox Mx1-Cre mice with or without PCL treatment on a morphological and molecular level, in terms of atherosclerosis and AAA development. AngII infusion in the study mice resulted in an increased atherosclerotic lesion area and increased infiltration of inflammatory leukocytes, which was not observed in mice with PCL induced prior to AngII infusion. This suggested that AngII infusion in this mouse model induced atherosclerosis development, and that plasma cholesterol levels represent a controlling factor. Furthermore, AngII infusion in $\mathrm{Ldlr}^{-/-} \mathrm{Apob}^{100 / 100} \mathrm{Mttp}{ }^{\text {flox/flox }} \mathrm{Mx1-Cre}$ mice caused a modest aneurysmal phenotype, and no differences in AAA development were observed between the different study groups. However, the fact that modest hypercholesterolemic mice did not develop AAA in a classical aneurysmal model indicated that plasma cholesterol levels are important for disease development.
\end{abstract}

Correspondence to: Ms Erika Folestad, Division of Vascular Biology, Department of Medical Biochemistry and Biophysics, Karolinska Institutet, Scheelesväg 2, 17177 Stockholm, Sweden E-mail: erika.folestad@ki.se

Key words: abdominal aortic aneurysm, cholesterol, angiotensin II, smooth muscle cell

\section{Introduction}

Abdominal aortic aneurysm (AAA) is a relatively common and potentially lethal disease. The most important risk factors associated with AAA include smoking, being male, age and family history (1-3). Coexisting atherosclerosis is a common feature in patients with AAA. Although AAA and atherosclerosis share certain common risk factors $(1,4)$, there are important histopathological and epidemiological differences that suggest they should be regarded as separate disease entities.

AAA is a relatively complex disease, and the mechanisms underlying the development and progression of the disorder remain to be completely understood. However, the main pathological hallmarks of established AAAs are chronic inflammation, vascular smooth muscle cell (vSMC) apoptosis, and extracellular matrix degradation in the media layer $(2,4)$.

The mechanisms driving atherosclerotic progression are characterized by a pathological build-up of plaque within the intimal layer, where endothelial dysfunction, proliferation of smooth muscle cells, macrophage infiltration and the accumulation of lipids serve central functions (5).

The involvement of atherosclerosis in aneurysm pathology is unclear. A common feature of potential interest is that AAA and atherosclerosis result in a clear inflammatory response (4). Hypercholesterolemia is associated with the development of atherosclerosis, but its association with AAA remains indistinct $(4,6)$. Studies using rodent models have suggested that treatment with plasma cholesterol lowering (PCL) statins attenuate aneurysmal growth in experimentally-induced AAAs $(7,8)$. However, results in humans are more conflicting. One previous study demonstrated that statins reduce the expression of selected inflammatory mediators within the aortic vessel wall, independent of their lipid lowering effects (9). Earlier clinical association studies indicated a potential growth inhibitory effect of statins on AAA, but this was not confirmed in larger, more recent studies (10-12).

The aim of the present study was to further investigate the association between AAA and atherosclerosis and, more specifically, how PCL treatment affects the development of AAA in

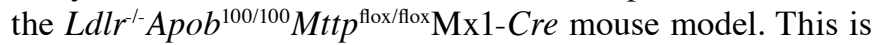


an atherosclerotic mouse model that has a plasma cholesterol profile similar to that of human hypercholesterolemia, with plasma cholesterol levels of $300-400 \mathrm{mg} / \mathrm{dl}$ on a regular chow diet, and develops atherosclerosis along the whole aortic tree. Furthermore, $\mathrm{Ldlr}^{-/} \mathrm{Apob}^{100 / 100} \mathrm{Mttp}{ }^{\text {flox/flox }} \mathrm{Mx} 1-\mathrm{Cr}$ mice have a genetic switch that allows lowering of plasma cholesterol levels at any desirable time point $(13,14)$. Previous studies in $L d l r^{-1-} A p o b^{100 / 100} M t t p^{\text {flox/flox }}$ Mx 1-Cre mice revealed that PCL treatment resulted in complete regression in mice with early atherosclerotic lesions, but was incomplete in mice with mature and advanced atherosclerotic lesions (13). Infusion of Angiotensin II (AngII) into mice is a well-established method to induce AAA. Aneurysm development has been reported to be markedly lower in AngII-treated wild-type C57BL/6 (non-hyperlipidemic) mice compared with hyperlipidemic mice, including $L_{d l r^{-/}}$or $a p o E^{-/}$mice (15). This suggests that hyperlipidemia may be of importance for AngII-induced AAA. Ldlr- $^{-1}$ Apo $b^{100 / 100} M t t p^{\text {flox/flox }} \mathrm{Mx1-Cre}$ mice have not previously been used in studies on AAA formation. Therefore, AAA was induced in $L d l r^{-/} A p o b^{100 / 100} M t t p^{\text {flox/flox }} \mathrm{Mx} 1-C r e$ mice $(14,16)$ by AngII-infusion to investigate its morphological and molecular effects on atherosclerosis and AAA establishment, as well as the effect of PCL therapy preceding AngII-infusion.

\section{Materials and methods}

Ethics statement. The present study was performed in accordance with Swedish legislation of animal welfare. The protocol for the present study was approved by the Stockholm North Ethical Committee on Animal Research (ethical permit no. N223/14). All animals used in the present study were monitored daily by the animal facility staff. Whenever illness was observed or suspected, animals were assessed according to the Karolinska Institutet extended assessment checklist for small rodents and rabbits, and euthanized if a total score of 0.3 was reached $(17,18)$. Animals were kept in a specific pathogen-free animal facility with a controlled temperature of $22^{\circ} \mathrm{C}$ and $12 / 12 \mathrm{~h}$ day-night cycle lightning. They were communally housed in standard cages with enrichment in the form of nesting material, and had ad libitum access to food and water.

Animals. Male Ldlr-/-Apo $b^{100 / 100}{ }^{-1 t} p^{\text {flox/flox } M x 1-C r e ~}$ (ID 004192-Mttptm2Sgy Ldlrtm1Her Apobtm2Sgy $\operatorname{Tg}(\mathrm{Mx} 1-\mathrm{cre}) 1 \mathrm{Cgn} / \mathrm{J})$ were obtained from the Jackson Laboratory for breeding (Bar Harbor, ME, USA; www.jax. org). All experiments were performed in mice bred in Scheele Animal Facility at Karolinska Institute. Mice were kept in standard cages with enrichment in form of nesting material and had free access to food/water. A total number of 75 mice were divided in three different groups and used in the study. Mice used had a weight of 20-25 g at the start of experiment. PCL was performed in 19-week-old mice by intraperitoneal injection (ip) of $200 \mu \mathrm{l}$ polyinosinic-polycytidylic acid (pI-pC; $1 \mu \mathrm{g} / \mu \mathrm{l}$, Invitrogen; Thermo Fisher Scientific, Inc., Waltham, MA, USA) every other day for a week to induce Cre expression and thereby Mttp recombination $\left(M t t p^{\Delta / \Delta}\right)$. Littermate controls received PBS (Mttp flox/flox). To induce aneurysm formation, micro-osmotic pumps (model 1004; Alzet; Durect Corporation, Cupertino, CA, USA) containing AngII (1,000 ng kg-1 min-1; Sigma-Aldrich; Merck KGaA, Darmstadt, Germany) or saline
(PBS; $0.9 \% \mathrm{NaCl}$ ) were implanted subcutaneously into the back of the mice one week after PCL treatment under isoflurane-induced anesthesia (2\%; Baxter Healthcare Corporation; Deerfield, IL, USA). One dose of buprenorphine (Temgesic; $10 \mu \mathrm{g} / \mathrm{ml}$ ) was given subcutaneously as an analgesic prior to surgery.

To determine whether $L d l r^{-/} A p o b^{100 / 100} M t t p p^{\text {flox/flox }} \mathrm{Mx1-Cre}$ mice developed AAA following AngII-infusion, several different starting points (including the age of mice) were investigated, as well as the duration of the AngII-infusion. In brief, AngII-containing pumps were implanted at 8 or 20 weeks of age for a period of 4 or 8 weeks. The group with the pump implanted at 20 weeks of age for 8 weeks was selected for further PCL experiments based on the degree of vascular remodeling following AngII-infusion.

Animals were monitored for any sign of pain. Blood from the tail vein or heart was collected in heparin-coated tubes prior to and following PCL treatment, and at the point of sacrifice. The osmotic pump was implanted at 20 weeks of age for 8 weeks (4+4). The study mice were sub-divided in 3 groups (n=9-12/group) as follows: Group 1, mice administered with PBS ip one week prior to implantation of pumps containing saline (Control, Ctrl); Group 2, mice administered with PBS ip one week prior to implantation of pumps containing AngII (AngII); and Group 3, mice administered with pI-pC one week prior to implantation of pumps containing AngII (AngII+PCL). Following the AngII-infusion period, mice were sacrificed by placing them in a chamber connected to a $\mathrm{CO}_{2}$ gas bottle and the lowest possible flow rate of $\sim 1 \mathrm{~L} / \mathrm{min}$ was used. The container was slowly filled with $\mathrm{CO}_{2}$ gas until asphyxiation of the mice. Mice were confirmed dead by assessing lack of heart beats for several minutes, after which the chest cavity was opened and thereafter blood was collected from cardiac puncture and then the right ear of the heart was cut. Aortas were collected.

Plasma analysis. Total plasma cholesterol and triglycerides were measured with the following colorimetric assays: Infinity Triglyceride,(Referenceno.TR22421; ThermoFisher Scientific, Inc.) and Infinity Cholesterol (Reference no. TR13421; Thermo Fisher Scientific, Inc.), using a POLARstar Omega plate reader (BMG Labtech GmbH, Ortenberg, Germany) according to the manufacturer's protocol. Plasma glucose levels were measured in non-starved mice using plasma taken from either the tail vein or the heart, with a Bayer Contour XT glucose meter and glucose test strips (Bayer AG, Leverkusen, Germany). The measured glucose levels were converted from $\mathrm{nmol} / \mathrm{l}$ to $\mathrm{mg} / \mathrm{dl}$ with the conversion factor $1 \mathrm{mmol} / \mathrm{l}=18,018 \mathrm{mg} / \mathrm{dl}$.

Immunohistological staining and analysis of the suprarenal aorta. Some suprarenal aorta samples were collected in $4 \%$ zinc formaldehyde fixative solution (Histolab Products AB, Spånga, Sweden) in room temperature for $24 \mathrm{~h}$ and then changed into PBS until embedded in paraffin, sectioned (5 $\mu \mathrm{m})$ and rehydrated using a descending alcohol series (X-tra Solv, 99, 95 and 70\% ethanol). The rest of the aortas were collected in 4\% paraformaldehyde (PFA; Sigma-Aldrich; Merck KGaA). PFA aortas were cleaned, cut into longitudinal sections, pinned out on black wax plates and stained with Sudan IV (5 g/l Sigma-Aldrich; Merck KGaA) for $6 \mathrm{~min}$ at room 
temperature. Images of sections were captured using a light microscope Nikon SMZ1000 microscope (Nikon Corporation, Tokyo, Japan) and quantified using an AxioGraphic station (Carl Zeiss AG, Oberkochen, Germany). Lesion area was calculated as the percentage of the total aortic area (the whole aortic tree from the aortic root until directly before the point where the iliac branches start). Antigen retrieval was performed using Diva Decloaker, 20X (Biocare Medical, LLC, Paheco, CA, USA) according to the manufacturer's protocol, endogenous peroxidase activity was blocked with $3 \%$ hydrogen peroxide, and sections were subsequently incubated in 5\% goat serum solution (Vector Laboratories, Burlingame, CA, USA) $1 \mathrm{~h}$ in room temperature. Then, sections were incubated with primary antibodies against mouse cluster of differentiation (CD)68 (1:500; cat. no. MCA1957; AbD Serotec; Bio-Rad Laboratories, Inc., Hercules, CA, USA) and transgelin (SM22 $\alpha ; 1: 4,000$, cat. no. ab14106; Abcam, Cambridge, UK) overnight at $4^{\circ} \mathrm{C}$. Sections were washed in PBS-Tween $(0.05 \%)$ $2 \times 10 \mathrm{~min}$. Thereafter, sections were incubated with secondary biotinylated rabbit anti-rat immunoglobulin $\mathrm{G}(\mathrm{IgG})(1: 500$; cat. no. BA-4001; Vector Laboratories, Ltd., Peterborough, UK) (CD68) or goat anti-rabbit IgG antibodies (1:500; cat. no. BA-1000; Vector Laboratories, Ltd., Peterborough, $\mathrm{UK})(\mathrm{SM} 22 \alpha) 1 \mathrm{~h}$ in room temperature. Avidin-biotin complexes (from the VECTASTAIN Elite ABC HRP kit; cat. no. PK-6100; Vector Laboratories, Ltd.) were added according to the manufacturer's protocol, and this was followed by staining with a DAB Peroxidase (HRP) Substrate kit (with nickel; cat. no. SK-4100; Vector Laboratories, Ltd.) according to the manufacturer's protocol. Sections were counterstained with QS hematoxylin (Vector Laboratories, Ltd.), visualized using a light microscope (AxioObserver, Carl Zeiss AG) and quantified with an AxioGraphic station (Carl Zeiss AG). For collagen staining, sections were incubated in Bouin's solution (Sigma-Aldrich; Merck KGaA) overnight, counterstained with Weigert's hematoxylin (Histolab Products AB), stained with a Masson Trichrome kit (Sigma-Aldrich; Merck KGaA) at room temperature and washed in $1 \%$ acetic acid according to the manufacturer's protocol. Mounted slides were visualized using a AxioCam ICc 1 light microscope (Carl Zeiss AG). Quantification and scoring of collagen and elastin was performed in whole aortic section and 3 whole sections per mouse were averaged. Two different blinded and experienced scientists performed the scoring of sections, in case of disagreement their results were averaged. The images were captured at x10 and x40 magnification. A scoring from 1 to 4 was then assigned, as follows: 1, a low amount of collagen in all layers of the aortic wall; 2, a low amount of collagen in the media or in the adventitia, 3, a high amount of collagen in the media or in the adventitia; and 4, a high amount of collagen in all layers of the aortic wall. Elastin degradation was quantified from the Masson Trichrome stained sections, and a scoring system from 1 to 4 was assigned to assess the variation in the degree of elastin degradation as follows: 1, intact elastin; 2, a low degree of degradation; 3 , intermediate degradation; and 4 , a high degree of degradation. Substantial vascular remodelling was defined as a $>50 \%$ increase in medial thickness compared with baseline values, which were defined as the average medial thickness from the control group mice. AAA was defined as a $>50 \%$ increase in the diameter of the aorta compared with baseline values, which were defined as the average of the aortic diameter from the control group mice.

Gene expression analysis. Suprarenal aortic samples were dissected, cleaned and stored in RNAlater (Ambion; Thermo Fisher Scientific, Inc.) at $80^{\circ} \mathrm{C}$. RNA was extracted using TRIzol reagent (Invitrogen; Thermo Fisher Scientific, Inc.), homogenized with a FastPrep instrument (MP Biomedicals, LLC, Santa Ana, CA, USA) and purified with an RNeasy Mini kit with a DNase1 treatment step (Qiagen, Inc., Valencia, CA, USA). RNA quantity was determined using a Nanodrop spectrometer (Thermo Fisher Scientific, Inc.). RNA was reversed transcribed using Superscript III First-strand Synthesis SuperMix (Invitrogen; Thermo Fisher Scientific, Inc.) according to the manufacturer's protocol, and cDNA was amplified using quantitative polymerase chain reaction (qPCR) analysis using TaqMan Fast Universal PCR Master Mix (Applied Biosystems; Thermo Fisher Scientific, Inc.) in a 7300 Real-Time PCR System according to the manufacture's protocol (Applied Biosystems; Thermo Fisher Scientific, Inc.). TaqMan Assay kits (Applied Biosystems; Thermo Fisher Scientific, Inc.) containing primers and probes were used for the following genes: Cd68 (Mm00839636_g1), filamin A (Flna; Mm01187533_m1), matrix metalloproteinase (Mmp)12 (Mm00500554_m1), Mmp9 (Mm00600163_m1), Cd3 (Mm00599683_m1), collagen type I a 1 chain (Collal; Mm00801666_m1) and Mast cell protease 9 (Mcpt9; Mm00775987_g1), and gene expression values were normalized to the house-keeping gene TATA-box binding protein (Mm00446973_m1). Samples were analyzed in duplicate using the standard curve method following the applied biosystems protocol (19).

En face analysis of aortas. Aortic arches collected in 4\% PFA were dissected, cleaned, cut into longitudinal sections and pinned out on black wax plates as previously described (20) stained with Sudan IV (Sigma-Aldrich; Merck KGaA), photographed with a light Nikon SMZ1000 microscope (Nikon Corporation) and quantified using an AxioGraphic station (Carl Zeiss AG). Lesion area was calculated as the percentage of the total aortic arch surface.

Blood pressure measurements. Blood pressure was recorded in isoflurane-anesthetized mice at the end of the experiment, one day prior to sacrifice, with a CODA Non-Invasive Blood Pressure System (Kent Scientific Corporation, Torrington, CT, USA) using an Occlusion tail cuff and a Volume Pressure Recorder sensor tail cuff, as previously described (21). Mice were anaesthetized for $15 \mathrm{~min}$ under $1.5 \%$ isoflurane, and were maintained at $35^{\circ} \mathrm{C}$ during the procedure. A total of 10 measurements were recorded following an acclimation period of 10 cycles.

Statistical analysis. The data were analyzed using GraphPad Prism software (version 7.0; GraphPad Software, Inc., La Jolla, CA, USA). Results are presented as the mean \pm standard error of the mean. Data analysis was accomplished using one-way analysis of variance with Tukey's post hoc analysis, or Kruskal-Wallis tests followed by Dunn's multiple comparisons test. $\mathrm{P}<0.05$ was considered to indicate a statistically significant difference. 

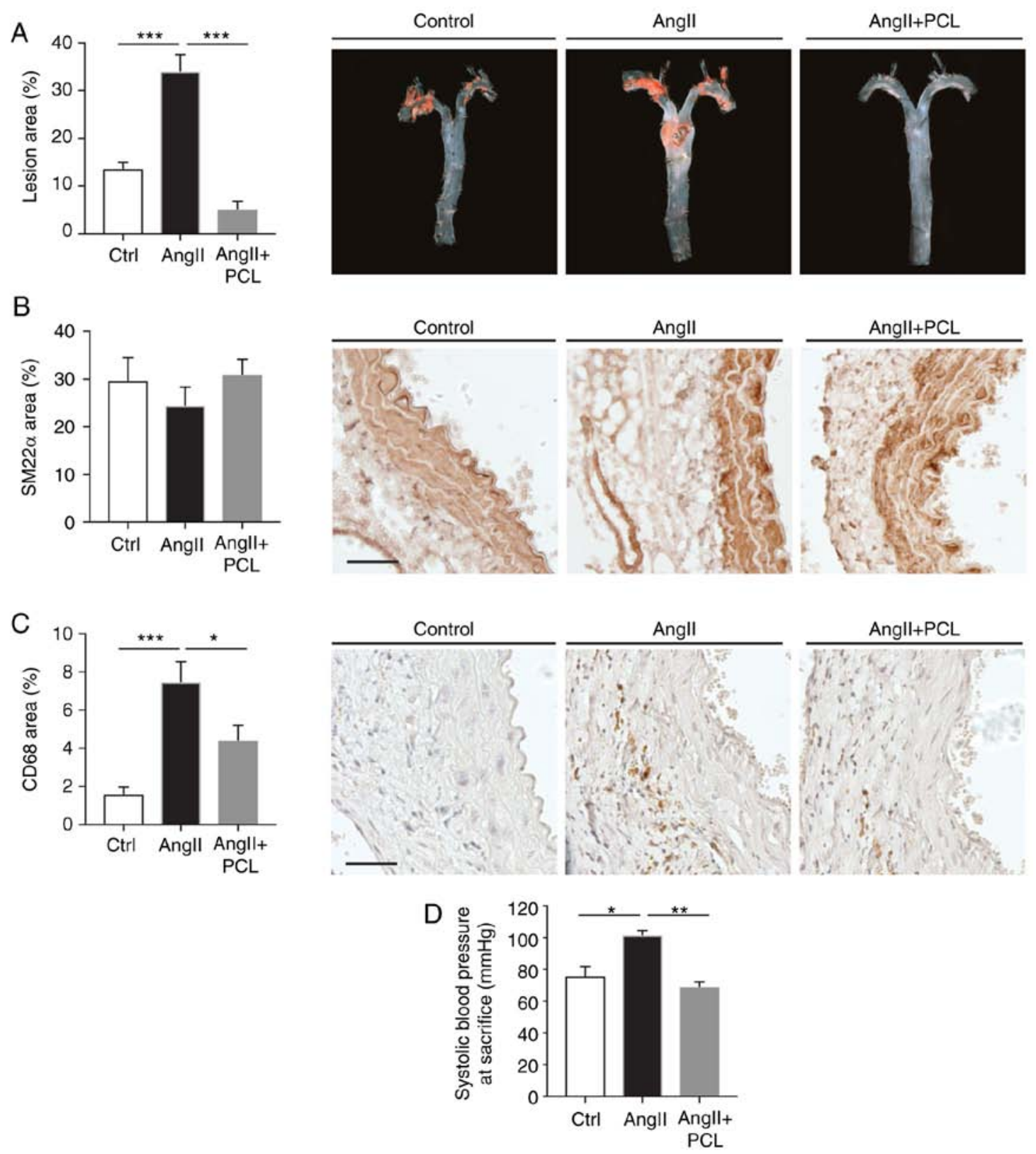

Figure 1. Atherosclerotic lesion characterization of AngII-infused $L d l r^{-1} A p o b^{100 / 100} M t t p^{A 0 x / f o x} M x 1$-Cre mice. (A) Percentage of lesion area, quantified by Sudan IV staining of the total aorta (left). Representative images of the total aortic tree magnified at $\mathrm{x} 0.8$ at the dissection microscope, for Ctrl $(\mathrm{n}=5)$, AngII $(\mathrm{n}=5)$ and AngII+PCL ( $\mathrm{n}=5$ ) mice. (B) Percentage of SM22 $\alpha$ stained area of total aortic root cross-sections (left) and representative images (right) for the quantified immunohistochemical staining (Ctrl, AngII, AngII+PCL; n=6-11). (C) Percentage of CD68 stained area of total aortic root cross-sections (left) and representative images (right) for the quantified immunohistochemical staining (Ctrl, AngII, AngII+PCL; n=6-11). (D) Systolic blood pressure (Ctrl, AngII, AngII+PCL; $\mathrm{n}=3$ ). Statistical significance was analyzed by one-way analysis of variance with Tukey's post hoc test, and data are presented as the mean \pm standard error of the mean. Scale bars represent $50 \mu \mathrm{m} .{ }^{*} \mathrm{P}<0.05$ and ${ }^{* * * *} \mathrm{P}<0.001$, with comparisons indicated by lines. AngII, angiotensin II; PCL, plasma cholesterol lowering; Ctrl, control.

\section{Results}

Atherosclerosis development in AngII-infused $\mathrm{Ldlr}^{-1-}$ Apo $\mathrm{b}^{100 / 100} \mathrm{Mttp} \mathrm{flox}^{\text {flox }} \mathrm{Mxl-Cre}$ mice. Baseline plasma profiles (cholesterol, triglycerides and glucose) were similar for the three different study groups (Ctrl, AngII and AngII+PCL; Table I). As expected, the plasma levels of cholesterol (+32\%), triglycerides $(+63 \%)$ and glucose $(+38 \%)$ were increased in the control group compared with baseline values. Infusion of AngII for eight weeks increased the plasma cholesterol content $(+30 \%)$ and the glucose $(+30 \%)$ levels in a similar manner to the control group. However, infusion of AngII increased the plasma triglyceride content by $+100 \%$ as compared with baseline values; a more pronounced increase than that in the control group. In PCL-treated animals, AngII infusion resulted in significantly decreased plasma cholesterol (-80\%) levels, unchanged triglyceride content and a minor increase in the amount of glucose $(+10 \%)$ as compared with the baseline levels (Table I). Control and AngII-infused mice had markedly increased plasma cholesterol, triglyceride and glucose levels when compared with starting values, which was not observed in animals with PCL treatment prior to AngII infusion. These results demonstrated that typical plasma parameters known to be regulated in atherosclerosis increased following AngII infusion in the mice without PCL. 
Table I. Plasma measurements of the difference mouse study groups.

\begin{tabular}{llccccc}
\hline Treatment & $\begin{array}{c}\text { CHL start } \\
(\mathrm{mg} / \mathrm{dl})\end{array}$ & $\begin{array}{c}\text { CHL sacrifice } \\
(\mathrm{mg} / \mathrm{dl})\end{array}$ & $\begin{array}{c}\text { TG start } \\
(\mathrm{mg} / \mathrm{dl})\end{array}$ & $\begin{array}{c}\text { TG sacrifice } \\
(\mathrm{mg} / \mathrm{dl})\end{array}$ & $\begin{array}{c}\text { Glucose start } \\
(\mathrm{mg} / \mathrm{dl})\end{array}$ & $\begin{array}{c}\text { Glucose sacrifice } \\
(\mathrm{mg} / \mathrm{dl})\end{array}$ \\
\hline Control & $163 \pm 10.1$ & $216 \pm 14.2^{\mathrm{a}}$ & $36.6 \pm 5.1$ & $59.7 \pm 3.8^{\mathrm{b}}$ & $209 \pm 8.3$ & $288 \pm 9.1^{\mathrm{b}}$ \\
AngII & $173 \pm 8.3$ & $225 \pm 14.3^{\mathrm{b}}$ & $40.5 \pm 3.4$ & $82.2 \pm 6.3^{\mathrm{b}, \mathrm{d}}$ & $207 \pm 7.7$ & $268 \pm 12.1^{\mathrm{b}}$ \\
AngII+PCL & $178 \pm 11.7$ & $36.7 \pm 4.1^{\mathrm{b}, \mathrm{c}, \mathrm{d}}$ & $34.9 \pm 2.8$ & $36.2 \pm 2.4^{\mathrm{c}, \mathrm{d}}$ & $201 \pm 5.8$ & $224 \pm 12.2^{\mathrm{a}}$ \\
\hline
\end{tabular}

${ }^{a} \mathrm{P}<0.05$ and ${ }^{\mathrm{b}} \mathrm{P}<0.001$ vs. starting value. ${ }^{\mathrm{c}} \mathrm{P}<0.001$ vs. control group. ${ }^{\mathrm{d}} \mathrm{P}<0.001$ vs. AngII group. Data are presented as the mean \pm standard error of the mean. There were no significant differences between the groups at the beginning of the present study. PCL, plasma cholesterol lowering; AngII, angiotensin II; CHL, cholesterol; TG, triglycerides.

A

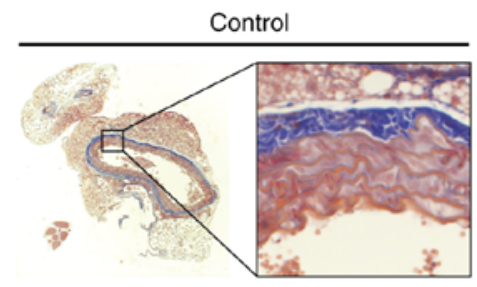

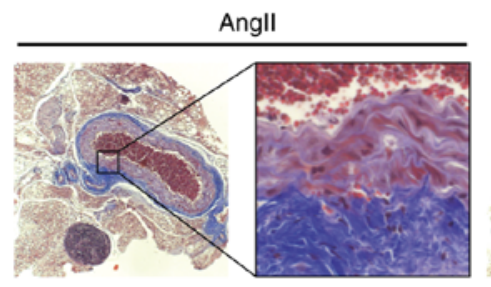
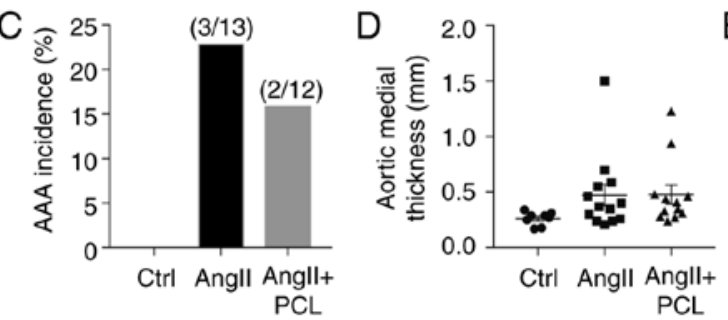

Angll+PCL
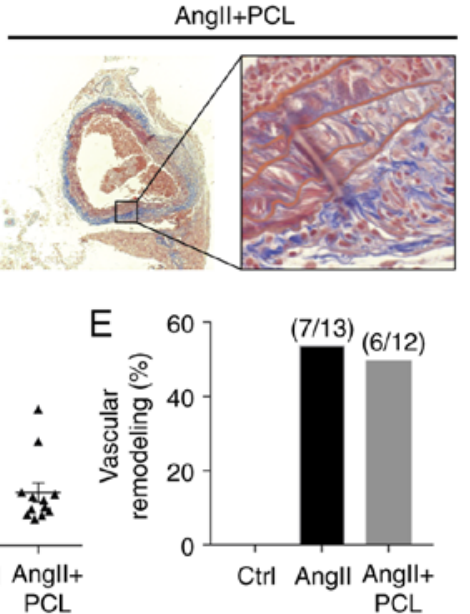
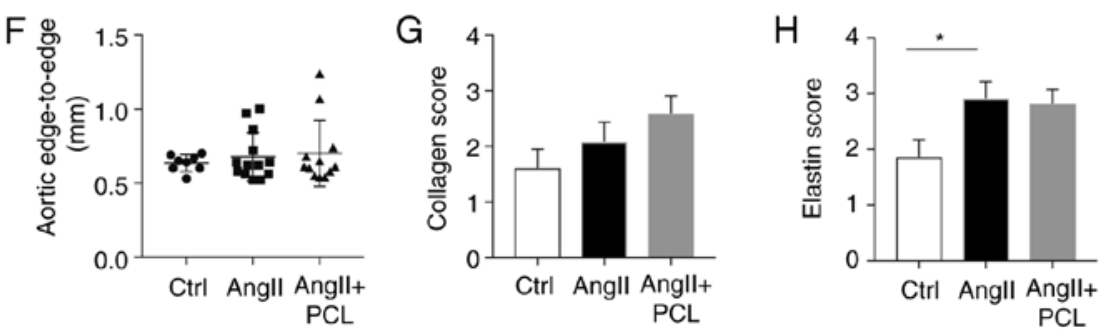

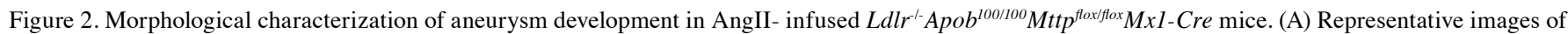
histological analyses magnified at x10 and x40, performed by Masson Trichrome staining in Ctrl (n=7), AngII (n=13) and AngII+PCL (n=12) mice. (B) The aortic outer diameter. (C) AAA incidence. (D) Medial thickness of the aorta. (E) Vascular remodeling. (F) Aortic edge-to-edge measurement. (G) Scoring of collagen content, as assessed with Masson Trichrome staining (Ctrl, n=7; AngII, n=11; AngII+PCL, n=10). (H) Scoring of elastin degradation (Ctrl, n=7; AngII, $\mathrm{n}=13$; AngII+PCL, $\mathrm{n}=12$ ). Substantial vascular remodeling was defined as a $>50 \%$ increase in medial thickness. Abdominal aortic aneurysm was defined as a $>50 \%$ increase in the diameter of the aorta. Statistical significance was analyzed by Kruskal-Wallis tests, with Dunn's multiple comparisons test. Data are presented as the mean \pm standard error of the mean. ${ }^{*} \mathrm{P}<0.05$, with comparisons indicated by lines. AngII, angiotensin II; PCL, plasma cholesterol lowering; VR, vascular remodeling; Ctrl, control.

Consistent with the plasma measurements, PCL pretreatment was demonstrated to reduce the development of atherosclerosis compared with non-treated, AngII-induced animals, which developed a similar atherosclerotic lesion area to the control mice (Fig. 1A). Immunohistological staining revealed that the smooth muscle cell marker SM22 $\alpha$ remained largely unaffected in the different study groups (Fig. 1B). CD68 staining for cellular infiltration of inflammatory leukocytes was significantly increased in non PCL-treated AngII-infused mice compared with mice with PCL pretreatment (Fig. 1C). Furthermore, measuring systemic systolic blood pressure revealed that AngII-infused animals without PCL pretreat- ment exhibited significantly higher blood pressure than mice with PCL (Fig. 1D).

Taken together, these results indicated that $L d l r^{-1-} A p o b^{100 / 100}$ $M t t p^{\text {flox/flox } M x 1-C r e ~ m i c e ~ i n f u s e d ~ w i t h ~ A n g I I ~ d e v e l o p e d ~ d i s t i n-~}$ guishable atherosclerosis, as compared with control animals or mice with PCL prior to AngII-infusion.

AAA development in AngII-infused Ldlr-/-Apob ${ }^{100 / 100}$ Mttp floxfflox Mxl-Cre mice. AAA development was monitored in the $\mathrm{Ldlr}^{-/} A p o b^{100 / 100} \mathrm{Mttp}{ }^{\text {flox/flox }} \mathrm{Mx} 1-\mathrm{Cr}$ e mice (Fig. 2). After 8 weeks of AngII-infusion, the increase in aortic diameter was modest and did not differ significantly between the three 

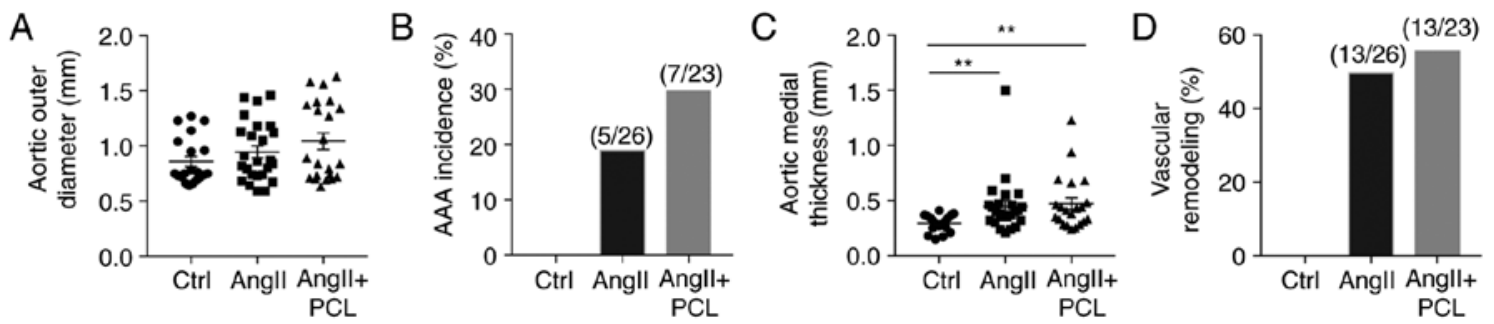

Figure 3. Morphological characterization of aneurysm development in AngII-infused $L d l r^{-1} \mathrm{Apob}^{100 / 100} \mathrm{Mttp}^{f 10 x / f o x} \mathrm{Mxl}$-Cre mice, including results from the pilot study. Different aortic measurements from Masson Trichrome staining at different time points from all animals used, including those from the pilot study. (A) Aortic outer diameter. (B) AAA incidence. (C) Medial thickness of the aorta. (D) Vascular remodeling. The cohort included mice at 20 weeks of age receiving AngII for 4 weeks (Ctrl, n=3; AngII, n=2; AngII+PCL, n=1) or mice at 8 weeks of age receiving AngII for 4 (Ctrl, n=6; AngII, n=7; AngII+PCL, $\mathrm{n}=7$ ) or 8 weeks (Ctrl, $\mathrm{n}=5$; AngII, $\mathrm{n}=4$; AngII+PCL, $\mathrm{n}=3$ ). Substantial vascular remodeling was defined as a $>50 \%$ increase in medial thickness. Abdominal aortic aneurysm was defined as a $>50 \%$ increase in the diameter of the aorta. Statistical significance was analyzed by Kruskal-Wallis tests with Dunn's multiple comparisons test. Data are presented as the mean \pm standard error of the mean. ${ }^{* *} \mathrm{P}<0.01$, with comparisons indicated by lines. AngII, angiotensin II; PCL, plasma cholesterol lowering; VR, vascular remodeling; Ctrl, control.
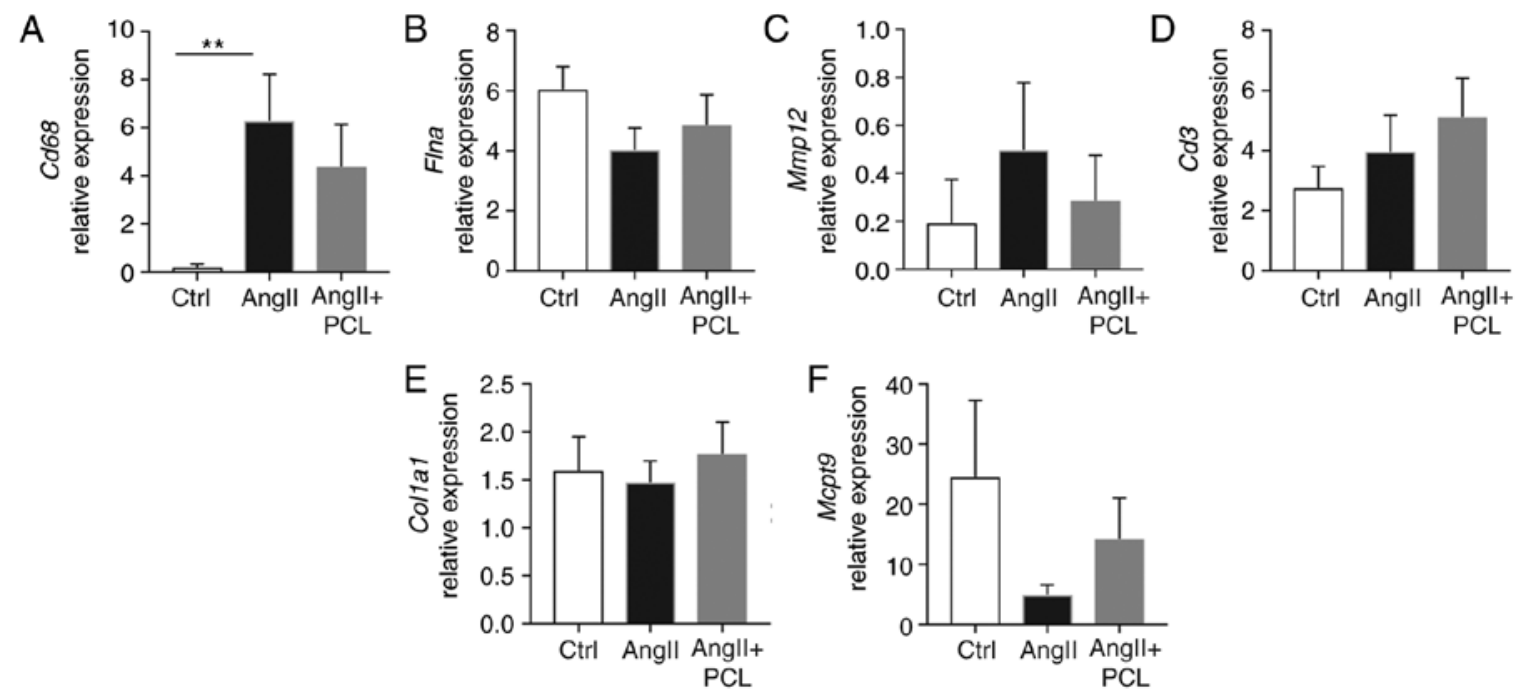

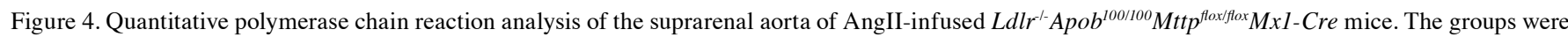
as follows: Ctrl (n=7), AngII, (n=11) and AngII+PCL (n=10). Gene expression levels of (A) Cd68, (B) Flna, (C) Mmp12, (D) Cd3, (E) Colla1 and (F) Mcpt9; all normalized to TATA-box binding protein. Data are presented as the mean \pm standard error of the mean. Statistical significance was analyzed by Kruskal-Wallis tests with Dunn's multiple comparisons test. ${ }^{* *} \mathrm{P}<0.01$, with comparisons indicated by lines. AngII, angiotensin II; PCL, plasma cholesterol lowering; Ctrl, control; $C d$, cluster of differentiation; Flna, filamin A; Mmp12, matrix metalloproteinase 12; Colla1, collagen type I $\alpha 1$ chain; Mcpt 9 , Mast cell protease 9.

groups (Fig. 2A, B and F). A total of $17 \%$ of PCL-treated mice developed an aneurysmal phenotype, compared with $23 \%$ of non-treated AngII-induced mice (Fig. 2A-C). Medial thickness was slightly increased in the two AngII-infused mice groups compared with saline-infused control animals (Fig. 2A and D). Mice with and without PCL prior to AngII-infusion exhibited vascular remodeling in 50 and $54 \%$ of cases, respectively (Fig. 2A and E). Overall aneurysmal formation was low in the $\mathrm{Ldlr}^{-1} \mathrm{Apob}^{100 / 100} \mathrm{Mtt} p^{\text {flox/flox }} \mathrm{Mx1-Cre}$ mouse model, and in order to obtain a larger study group, all time points used in the pilot study were analyzed together with the 8 week study (Fig. 3). Data from the larger cohort revealed similar results.

Histological staining and scoring of collagen in cross sections of suprarenal aortas demonstrated a slight, non-significant increase of collagen production in the AngII-infused groups compared with the control animals (Fig. 2A and G). Further histological staining and scoring of elastin revealed small, but significant differences in elastin degradation in non PCL-treated AngII-infused mice, while no significant differ- ences were observed in PCL-treated mice prior to infusion of AngII, compared with control aortas (Fig. 2A and H).

To summarize, AngII-infusion in $L d l r^{-/-} A_{p o b} b^{100 / 100}$ $M t t p^{\text {flox/flox }} \mathrm{Mx} 1-\mathrm{Cr}$ mice, a mouse model with moderately increased cholesterol levels, resulted in a low incidence of AAA that was unaffected by PCL treatment.

Gene expression analysis in AngII-infused $\mathrm{Ldll}^{-1} \mathrm{Apob}^{100 / 100} \mathrm{Mttp}^{\text {floxfflox } M x 1-C r e ~ m i c e . ~ G e n e ~ e x p r e s-~}$ sion levels of the macrophage marker $C d 68$ were markedly elevated in the non-treated AngII-infused group, and this was not observed to the same degree in the PCL-pretreated mice since the difference was non-significant compared with the control (Fig. 4A). The gene Flna, encoding the non-contractile cytoskeletal protein filamin A, was decreased in non-pretreated, AngII-infused mice as compared with control and PCL treated animals (Fig. 4B), indicating loss of vSMC, since filamin A primarily binds to actin. Expression of Mmp12 is often elevated in AAA as a result of macro- 
phage infiltration (22). Mmp12 upregulation was observed in non-pretreated AngII-infused animals, but not in animals with PCL treatment prior to infusion of AngII, compared with the control (Fig. 4C). Other key AAA genes investigated, including Cd3, Collal and Mcpt9, were unaltered or exhibited changes that were not significant (Fig. 4D-F). In summary, the few alterations in gene expression and the large standard deviations in the different groups are potentially reflected by the low incidence of aneurysms. However, expression of several of the genes examined were altered in the AngII-infused group in a manner that was not observed in PCL-treated mice, indicating that plasma cholesterol levels affect disease development. Furthermore, consistent with data observed from immunhistological staining, Cd68 was upregulated in AngII-infused mice, but this was not observed in PCL-treated mice.

\section{Discussion}

In the present study, the morphological and molecular effects of PCL therapy on atherosclerosis and aneurysmal formation were evaluated in an atherosclerotic AAA mouse model. The $\mathrm{Ldlr}^{-/} \mathrm{Apob}^{100 / 100} \mathrm{Mttp}{ }^{\text {flox/flox }} \mathrm{Mx1-Cre}$ mouse model is an atherosclerotic model with a moderate increase in plasma cholesterol levels, similar to those observed in human hypercholesterolemia. This mouse model has not previously been used to examine the effects of AngII-infusion on atherosclerotic development or aneurysmal formation.

The main finding of the present study was that AngII-infusion increased plasma cholesterol and triglyceride levels, as well as atherosclerotic development. Notably, AngII-induced atherosclerosis was prevented by PCL treatment prior to infusion of AngII, which is in agreement with previous studies on the hyperlipidemic apoe a- $^{-}$mouse model $(23,24)$. This suggests that the renin-AngII system is mechanistically involved in the pathogenesis of atherosclerosis. In addition, the present study also demonstrated that the amount of plasma cholesterol is important for atherosclerosis progression following AngII-infusion, since PCL averts the progression of atherosclerosis development. Our group previously demonstrated that PCL slows and prevents the progression of atherosclerosis regardless of lesion stage, and may lead to complete regression if PCL is induced in mice with early lesions (13). Furthermore, an increase in the infiltration of inflammatory leukocytes and systemic blood pressure was observed in AngII-infused animals, and this was also reversed by PCL. AngII is known to be involved in the regulation of blood pressure and is a potent hypertensive agent. Certain previous publications support these results (25), while others have been unable to detect any increase in blood pressure following AngII-infusion (24).

Despite its distinct effect in atherosclerosis development, as well as its impact on the expression of genes known to be involved in AAA development, the relatively low incidence of AAA in the AngII-treated $L d l r^{-/} A p o b^{100 / 100} M t t p^{\text {flox/flox }} \mathrm{Mx1-Cre}$ mice is an important limitation of the model, where PCL pretreatment had no significant effect on aneurysm formation. In other common mouse models used to study AAA, including apoe $^{-/-}$mice and $\mathrm{Ldlr}^{-/-}$mice on a high-fat diet, the occurrence of AAA formation following AngII infusion ranged from $70-80 \%$, compared with only $20-30 \%$ observed in our model $(26,27)$. This relatively low AAA incidence makes it difficult to determine the significance of the differences observed in the limited number of mice. However, including experiments from a pilot study and almost doubling the number of mice elicited similar results.

One explanation for the low rate of aneurysmal formation may be that the mice used in the present study were modestly hypercholesterolemic $(200 \mathrm{mg} / \mathrm{dl})$ compared with other models commonly used to study AAA, where plasma cholesterol levels reach $1,500 \mathrm{mg} / \mathrm{dl}$ (28). In a parallel study using the same batch of AngII in apoe $e^{-/-}$mice, AAA occurred in $75 \%$ of the mice (data not shown). Liu et al demonstrated that hypercholesterolemia increased the development of AngII-induced AAA in mice (28), indicating that higher cholesterol levels are required for AAA formation. Furthermore, induction of aneurysm in normocholesterolemic $\mathrm{C} 57 \mathrm{BL} / 6 \mathrm{~J}$ mice resulted in a low incidence rate of AAA, supporting the requirement of high cholesterol levels for aneurysmal development $(15,28)$.

Despite the lack of an overall effect on AAA formation, PCL pretreatment resulted in significantly decreased degradation of elastin. Elastin degradation has been suggested to be a key event in AAA formation $(1,4)$. In addition, on the molecular level, the expression of one key gene for AAA development, Flna, was decreased in AngII-infused mice, and this was not observed in mice with PCL pretreatment. This indicated that the degree of vSMC phenotypic modulation and loss of vSMCs (29) in mice with PCL prior to infusion of AngII was less pronounced, further strengthening the hypothesis that plasma cholesterol levels are involved in aneurysmal formation.

Historically, AAA has been considered to be an outcome of atherosclerosis. However, data obtained from several previous studies investigating human and mouse AAA models has suggested that, in the majority of cases, atherosclerosis merely co-exists with AAA pathology $(1,4)$. Taken together, the data obtained in the present study, where AngII-infusion was

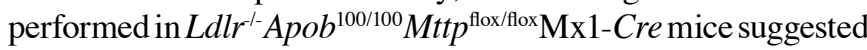
that atherosclerosis and AAA are two separate diseases, which is consistent with data from other previous studies $(1,4)$. The present study suggested that atherosclerosis and aneurysmal formation develop independently, but common factors, including plasma cholesterol levels, affect the two diseases. In addition, although it exerted a weak response on AAA formation, PCL treatment appeared to be of benefit. The fact that the mouse model used in the present study, which exhibited a modest increase in plasma cholesterol levels, had a low AAA occurrence rate. This indicated that plasma cholesterol levels are important for disease development. To fully address this issue, further studies in severe hypercholesterolemic mice are required.

\section{Acknowledgements}

Not applicable.

\section{Funding}

No funding was received.

\section{Availability of data and materials}

All data generated or analyzed during this study are included in this published article. 


\section{Authors' contributions}

AR contributed to the generation of the scientific hypothesis, study design, all animal experiments, immunohistrochemistry (IHC) studies regarding the superior part of the aortas (arch and root) for cryosectioning and staining, as well as for en face pinning and atherosclerosis analysis. She performed further paraffin-IHC of the suprarenal aortas and the gene expression analysis, as wel as all plasma measurements. She analysed all results and contributed to the manuscript composition. EV participated in the study design, performed part of the IHC studies regarding the suprarenal aortas in paraffin, contributed to the manuscript composition, gave feedback and revised it. AW participated in the study design, results' discussion and manuscript feedback and revision. TL participated in the discussion of the hypothesis, study design, data revision and interpretation. JS, EF and DW contributed to generate the hypothesis, the study design, analysed the results and contributed to the manuscript composition and revision.

\section{Ethics approval and consent to participate}

The protocol for the present study was approved by the Stockholm North Ethical Committee on Animal Research (ethical permit no. N223/14).

\section{Consent for publication}

Not applicable.

\section{Competing interests}

The authors declare that they have no competing interests.

\section{References}

1. Toghill BJ, Saratzis A and Bown MJ: Abdominal aortic aneurysm-an independent disease to atherosclerosis? Cardiovasc Pathol 27: 71-75, 2017.

2. Kuivaniemi H, Ryer EJ, Elmore JR and Tromp G: Understanding the pathogenesis of abdominal aortic aneurysms. Expert Rev Cardiovasc Ther 13: 975-987, 2015.

3. Wahlgren CM, Larsson E, Magnusson PK, Hultgren R and Swedenborg J: Genetic and environmental contributions to abdominal aortic aneurysm development in a twin population. J Vasc Surg 51: 3-7, 2010.

4. Peshkova IO, Schaefer G and Koltsova EK: Atherosclerosis and aortic aneurysm-is inflammation a common denominator? FEBS J 283: 1636-1652, 2016.

5. Tabas I, García-Cardeña G and Owens GK: Recent insights into the cellular biology of atherosclerosis. J Cell Biol 209: 13-22, 2015.

6. Golledge $J$ and Norman PE: Atherosclerosis and abdominal aortic aneurysm: Cause, response, or common risk factors? Arterioscler Thromb Vasc Biol 30: 1075-1077, 2010.

7. Steinmetz EF, Buckley C, Shames ML, Ennis TL, Vanvickle-Chavez SJ, Mao D, Goeddel LA, Hawkins CJ and Thompson RW: Treatment with simvastatin suppresses the development of experimental abdominal aortic aneurysms in normal and hypercholesterolemic mice. Ann Surg 241: 92-101, 2005.

8. Kalyanasundaram A, Elmore JR, Manazer JR, Golden A, Franklin DP, Galt SW, Zakhary EM and Carey DJ: Simvastatin suppresses experimental aortic aneurysm expansion. J Vasc Surg 43: 117-124, 2006.

9. van der Meij E, Koning GG, Vriens PW, Peeters MF, Meijer CA, Kortekaas KE, Dalman RL, van Bockel JH, Hanemaaijer R, Kooistra T, et al: A clinical evaluation of statin pleiotropy: Statins selectively and dose-dependently reduce vascular inflammation. PLoS One 8: e53882, 2013.
10. Takagi $H$, Yamamoto $H$, Iwata $K$, Goto $S$ and Umemoto $T$; ALICE (All-Literature Investigation of Cardiovascular Evidence) Group: Effects of statin therapy on abdominal aortic aneurysm growth: A meta-analysis and meta-regression of observational comparative studies. Eur J Vasc Endovasc Surg 44: 287-292, 2012.

11. Twine CP and Williams IM: Systematic review and meta-analysis of the effects of statin therapy on abdominal aortic aneurysms. Br J Surg 98: 346-353, 2011.

12. Wemmelund H, Hogh A, Hundborg HH, Thomsen RW, Johnsen SP and Lindholt JS: Statin use and rupture of abdominal aortic aneurysm. Br J Surg 101: 966-975, 2014.

13. Björkegren JL, Hägg S, Talukdar HA, Foroughi Asl H, Jain RK, Cedergren C, Shang MM, Rossignoli A, Takolander R, Melander O, et al: Plasma cholesterol-induced lesion networks activated before regression of early, mature, and advanced atherosclerosis. PLoS Genet 10: e1004201, 2014.

14. Rossignoli A, Shang MM, Gladh H, Moessinger C, Foroughi Asl H, Talukdar HA, Franzén O, Mueller S, Björkegren JL, Folestad E, et al: Poliovirus receptor-related 2: A cholesterol-responsive gene affecting atherosclerosis development by modulating leukocyte migration. Arterioscler Thromb Vasc Biol 37: 534-542, 2017.

15. Deng GG, Martin-McNulty B, Sukovich DA, Freay A, Halks-Miller M, Thinnes T, Loskutoff DJ, Carmeliet P, Dole WP and Wang YX: Urokinase-type plasminogen activator plays a critical role in angiotensin II-induced abdominal aortic aneurysm. Circ Res 92: 510-517, 2003.

16. Skogsberg J, Lundström J, Kovacs A, Nilsson R, Noori P, Maleki S, Köhler M, Hamsten A, Tegnér J and Björkegren J: Transcriptional profiling uncovers a network of cholesterol-responsive atherosclerosis target genes. PLoS Genet 4: e1000036, 2008.

17. Assessment of health status of small rodents and rabbits when illness is suspected. Journal, 2015.

18. Extended assessment of health status of small rodents and rabbits postoperatively or when necessary. Journal, 2015.

19. Larionov A, Krause A and Miller W: A standard curve based method for relative real time PCR data processing. BMC Bioinformatics 6: 62, 2005.

20. Veniant MM, Sullivan MA, Kim SK, Ambroziak P, Chu A, Wilson MD, Hellerstein MK, Rudel LL, Walzem RL and Young SG: Defining the atherogenicity of large and small lipoproteins containing apolipoprotein B100. J Clin Invest 106: 1501-1510, 2000.

21. Gladh H, Folestad EB, Muhl L, Ehnman M, Tannenberg P, Lawrence AL, Betsholtz $\mathrm{C}$ and Eriksson U: Mice lacking platelet-derived growth factor D display a mild vascular phenotype. PLoS One 11: e0152276, 2016.

22. Longo GM, Buda SJ, Fiotta N, Xiong W, Griener T, Shapiro S and Baxter BT: MMP-12 has a role in abdominal aortic aneurysms in mice. Surgery 137: 457-462, 2005.

23. Daugherty A and Cassis LA: Mouse models of abdominal aortic aneurysms. Arterioscler Thromb Vasc Biol 24: 429-434, 2004.

24. Daugherty A, Manning MW and Cassis LA: Angiotensin II promotes atherosclerotic lesions and aneurysms in apolipoprotein E-deficient mice. J Clin Invest 105: 1605-1612, 2000.

25. Cassis LA, Marshall DE, Fettinger MJ, Rosenbluth B and Lodder RA: Mechanisms contributing to angiotensin II regulation of body weight. Am J Physiol 274: E867-E876, 1998.

26. Qin Z, Bagley J, Sukhova G, Baur WE, Park HJ, Beasley D, Libby P, Zhang Y and Galper JB: Angiotensin II-induced TLR4 mediated abdominal aortic aneurysm in apolipoprotein E knockout mice is dependent on STAT3. J Mol Cell Cardiol 87: 160-170, 2015.

27. Thatcher SE, Zhang $X$, Howatt DA, Yiannikouris F, Gurley SB, Ennis T, Curci JA, Daugherty A and Cassis LA: Angiotensin-converting enzyme 2 decreases formation and severity of angiotensin II-induced abdominal aortic aneurysms. Arterioscler Thromb Vasc Biol 34: 2617-2623, 2014.

28. Liu J, Lu H, Howatt DA, Balakrishnan A, Moorleghen JJ, Sorci-Thomas M, Cassis LA and Daugherty A: Associations of ApoAI and ApoB-containing lipoproteins with AngII-induced abdominal aortic aneurysms in mice. Arterioscler Thromb Vasc Biol 35: 1826-1834, 2015.

29. Ailawadi G, Moehle CW, Pei H, Walton SP, Yang Z, Kron IL, Lau CL and Owens GK: Smooth muscle phenotypic modulation is an early event in aortic aneurysms. $\mathbf{J}$ Thorac Cardiovase Surg 138: 1392-1399, 2009. 